Asian J. Med. Biol. Res. 2018, 4 (4), 383-388; doi: 10.3329/ajmbr.v4i4.40111

\author{
Asian Journal of \\ Medical and Biological Research \\ ISSN 2411-4472 (Print) 2412-5571 (Online) \\ www.ebupress.com/journal/ajmbr
}

\title{
Article \\ Measurement of circulating insulin-like peptide 3 and testosterone concentrations in pre-pubertal, tropical male goats
}

\author{
Anuradha Wimalarathne ${ }^{1, a}$, Lakshitha Fonseka ${ }^{1, a}$, Indunil Pathirana ${ }^{1, *}$, Suranga Kodithuwakku ${ }^{2}$ and Noritoshi \\ Kawate $^{3}$ \\ ${ }^{1}$ Department of Animal Science, Faculty of Agriculture, University of Ruhuna, Kamburupitiya 81100, Sri Lanka \\ ${ }^{2}$ Department of Animal Science, Faculty of Agriculture, University of Peradeniya, Peradeniya 20400, Sri Lanka \\ ${ }^{3}$ Department of Advanced Pathobiology, Graduate School of Life and Environmental Sciences, Osaka \\ Prefecture University, Izumisano, Osaka 598-8531, Japan
}

*Corresponding author: Indunil Pathirana, Department of Animal Science, Faculty of Agriculture, University of Ruhuna, Kamburupitiya 81100, Sri Lanka. Phone: +94412292200; E-mail: indunilvet@ ansci.ruh.ac.lk

${ }^{\mathrm{a}}$ Contributed equally to this work

Received: 07 December 2018/Accepted: 22 December 2018/ Published: 30 December 2018

\begin{abstract}
The present study attempted to: (1) simplify the existing enzyme immunoassay (EIA) for the measurement of insulin-like peptide 3 (INSL3) concentrations in goats (2) to measure circulating INSL3 and testosterone in pre-pubertal Jamnapari X local crossbred goats (3) to examine the relationships among INSL3 concentration, testosterone concentration, scrotal circumference and body weight during the pre-pubertal age. Serial blood samples were collected from normal pre-pubertal male Jamnapari X local crossbred goats $(n=6)$ at the ages of 19 to 28 weeks. Serum INSL3 was measured using a recently reported EIA with modifications to the original procedure. The detection ranges of the INSL3 and testosterone assays were 0.08 to $80 \mathrm{ng} / \mathrm{mL}$ and 0.01 to $40 \mathrm{ng} / \mathrm{mL}$, respectively. The intra-assay coefficient of variations were $3.79 \%$ for INSL3 $(\mathrm{n}=6)$ and $3.72 \%(\mathrm{n}$ = 6) for testosterone. Serum INSL3 concentrations ranged from $13.62 \pm 3.25$ to $22.45 \pm 6.09 \mathrm{ng} / \mathrm{mL}$ (mean \pm SEM) in pre-pubertal goats. Those concentrations increased $(\mathrm{p}<0.05)$ from $20(13.62 \pm 3.25 \mathrm{ng} / \mathrm{mL})$ to 22 $(22.45 \pm 6.09 \mathrm{ng} / \mathrm{mL})$ weeks of age. Testosterone concentrations ranged from $0.30 \pm 0.07$ to $1.22 \pm 0.43 \mathrm{ng} / \mathrm{mL}$ in pre-pubertal goats. A significant drop was observed in testosterone concentrations at 23 weeks of age. INSL3 was correlated $(r=0.58 ; p<0.05)$ with scrotal circumference while no significant correlation was observed among other tested parameters. A rapid, sensitive EIA system was simplified to quantify INSL3 in goats, by simplifying the existing procedure. Different serum INSL3 and testosterone dynamics were found from 19 to 28 weeks of age of the goats. Compared with testosterone, INSL3 dynamics seemed to be more consistent with the age of pre-pubertal goats and showed a relationship with the testicular growth.
\end{abstract}

Keywords: enzyme immunoassay; INSL3; male goats; pre-pubertal; serum; testosterone

\section{Introduction}

Insulin-like peptide 3 (INSL3), along with testosterone, is a major secretory product of Leydig cells in mature testes of all male mammals (Ivell et al., 2013), including male goats (Siqin et al., 2013). This hormone plays an important role in reproductive physiology of male animals and humans (Bay and Andersson, 2011), and these functions include the initiation of transabdominal phase of testicular descend (Nef and Parada, 1999; Zimmermann et al., 1999) and suppression of germ cell apoptosis (Kawamura et al., 2004), as shown in experimental animals. Readily detectable levels of INSL3 have been reported in many mammalian species including humans (Anand-Ivell et al., 2006; Bay et al., 2005; Bullesbach et al., 1999; Ferlin et al., 2006; Wikstrom et al., 2006), dogs (Pathirana et al., 2012), cattle (Hannan et al., 2015; Kawate et al., 2011) and goats 
(Hannan et al., 2016; Hannan et al., 2017b), and peripheral concentrations of INSL3 changes with GnRH stimulation of LH release (Hannan et al., 2015; Hannan et al., 2016), age (Anand-Ivell et al., 2006; Bay et al., 2007; Pathirana et al., 2012) and testicular abnormalities (Bay et al., 2005; Pathirana et al., 2012). Therefore, it has been suggested that blood INSL3 concentrations can be used as a biomarker to assess the Leydig cell function. Furthermore, INSL3 has been used as a testis-specific biomarker for the assessment of pubertal development (Anand-Ivell et al., 2009; Ferlin et al., 2006; Johansen et al., 2014; Wikstrom et al., 2006). Due to differential patterns of regulation, the measurement of both INSL3 and testosterone in the same animal may provide an added benefit in assessing Leydig cell function in vivo. However, the concentrations of INSL3 in peripheral blood of goats in tropics are yet to be reported. The only available reports are on a native Japanese miniature goat breed, Shiba (Hannan et al., 2016; Hannan et al., 2017a; Hannan et al., 2017b). With this background, it is noteworthy to investigate the endocrine changes during the pre-pubertal age of male goats by measuring both circulating INSL3 and testosterone.

At present, various methods are used to quantify hormones in blood of many species. Among these, enzyme immunoassay (EIA) is a reliable, widely used biochemical technique. Plasma INSL3 concentrations of several species, i.e., dogs (Pathirana et al., 2012), cattle (Hannan et al., 2015; Kawate et al., 2011) and goats (Hannan et al., 2016; Hannan et al., 2017a) have been previously measured through immunoassays which include an INSL3 extraction step using a vacuum centrifuge. A simplified EIA protocol which requires less expensive equipment is important for the research studies conducted in developing countries with a resource-limited setup. The objectives of the present study were to (1) simplify the existing EIA protocol for the measurement of INSL3 concentrations in goats, (2) measure the circulating INSL3 and testosterone in pre-pubertal Jamnapari $\times$ local crossbred goats and (3) examine relationships among serum hormone (INSL3 and testosterone) concentrations, scrotal circumference and body weight in male goats at pre-pubertal age.

\section{Materials and Methods}

\subsection{Animals, body measurements and blood sampling}

Jamnapari $\times$ local crossbred male kids $(n=6)$ born on the same day in the Faculty Teaching Farm, Faculty of Agriculture, University of Ruhuna were used for this study. All kids were normal at birth and had no apparent abnormalities and remained healthy throughout the experiment. All goats were at 19 weeks of age at the beginning of the study. After restraining of the animals, scrotal circumference was measured at the age of 19, 22 and 28 weeks by using a flexible tape, and the body weight was also recorded. Serial blood samples (three to five milliliters) were collected at weeks $19,20,22,23$ and 28 from a jugular vein puncture. Serum was separated by following a $2000 \times g$ centrifugation for $20 \mathrm{~min}$ and was stored in microcentrifuge tubes at $-20{ }^{\circ} \mathrm{C}$ prior to hormone assays. The samples were thawed just prior to the INSL3 and testosterone assays. The protocol of the experiment was approved by the Research Ethics Committee of the Faculty of Agriculture, University of Ruhuna, Sri Lanka.

\subsection{INSL3 assay}

A competitive EIA was developed to quantify INSL3 by modifying the previously described procedures for goats (Hannan et al., 2016) and cattle (Kawate et al., 2011). Previously developed INSL3 EIA was modified by omitting the INSL3 extraction procedure which involved a vacuum centrifugation step of more than $3 \mathrm{~h}$. In brief, Strip wells were coated with $100 \mu \mathrm{L}$ of anti-mouse $\operatorname{IgG~}(5 \mu \mathrm{g} / \mathrm{mL}$ in $0.05 \mathrm{M}$ sodium bicarbonate; $\mathrm{pH}$ 9.7), and non-specific binding sites were blocked overnight with 2\% BSA, 0.02\% ProClin 950 in $0.01 \mathrm{M}$ PBS, pH 7.4 (assay buffer). Fifty-microliter of each standard or serum sample and $50 \mu \mathrm{L}$ of anti-bovine INSL3 (1:1,000,000 dilution in assay buffer; 2-8F (Bullesbach and Schwabe, 2002), a gift from Dr. E.E. Büllesbach, Medical University of South Carolina, USA) were then dispensed into the wells and incubated for $2 \mathrm{~h}$ at room temperature. Subsequently, $50 \mu \mathrm{L}$ of biotinylated human INSL3 peptide $(1 \mathrm{ng} / \mathrm{mL}$ in assay buffer) was added and incubated for a further $1 \mathrm{~h}$. The wells were washed three times with saline containing $0.05 \%$ Tween-20 and incubated for $30 \mathrm{~min}$ with horseradish peroxidase-labeled streptavidin $(100 \mathrm{ng} / \mathrm{mL}$ in assay buffer). Next, the wells were washed three times with saline containing $0.05 \%$ Tween-20 and were incubated for another $30 \mathrm{~min}$ with $100 \mu \mathrm{L}$ 3,3',5,5'-tetramethylbenzidine (TMB) substrate solution. The reaction was stopped by adding 100 $\mu \mathrm{L}$ of $2 \mathrm{M}$ sulfuric acid, and optical density was measured at $450 \mathrm{~nm}$ with a $630 \mathrm{~nm}$ reference using a microplate reader (UT-2100C, MRC, Israel).

\subsection{Testosterone assay}

Extraction of testosterone from goat serum was performed according to the procedure described previously (Kawate et al., 2011; Pathirana et al., 2012). For extracted standards or extracted samples, testosterone EIA 
using the HRP-labeled testosterone and anti-testosterone antibody was performed using a method described previously (Kawate et al., 2011; Pathirana et al., 2012). The minimum detection limit of the assay was 0.01 $\mathrm{ng} / \mathrm{mL}$, and detection was reliable in the range 0.01 to $40 \mathrm{ng} / \mathrm{mL}$. The intra-assay coefficient of variations was $3.72 \%(n=6)$ for testosterone.

\subsection{Statistical analysis}

Differences in mean INSL3 and testosterone concentrations among groups (age in weeks) were compared using pairwise comparisons of the generalized linear models (GZLM; SPSS version 20.0, IBM Corporation, Somers, NY, USA) procedure by the least significant difference (LSD) post hoc test. Correlations among hormone concentrations (INSL3 and testosterone), testicular circumference and body weight in pre-pubertal goats were estimated by using the Pearson correlation coefficient (IBM SPSS Statistics 20.0). Data were expressed as mean \pm SEM, with differences considered significant at $\mathrm{P}<0.05$.

\section{Results}

The existing EIA system (Hannan et al., 2016; Kawate et al., 2011) was simplified to measure circulating INSL3 in male goats, by omitting the extraction procedure which requires a vacuum centrifugation step. The removal of vacuum centrifugation step shortened the assay procedure for more than $3 \mathrm{~h}$. The minimum detection limit of the assay was $0.08 \mathrm{ng} / \mathrm{mL}$ and the percent binding $(\mathrm{B} / \mathrm{B} 0,96.8 \pm 1.6 \%)$ at this limit was significantly less $(\mathrm{P}<0.05)$ than $\mathrm{B} 0$, as observed in three consecutive standard curves. The sensitivity range of the assay was 0.08 to $80 \mathrm{ng} / \mathrm{mL}$ (Figure 1A). The simplified EIA system demonstrated a parallel drop in percent binding between INSL3 standards compared with a serially diluted (two to eight fold) serum sample from a male goat (Figure 1B). The intra-assay coefficient of variation was $3.79 \%(n=6)$ for INSL3 and the intra-assay variation observed was quite acceptable.

As measured by the present EIA system, serum INSL3 concentrations ranged from $13.62 \pm 3.25$ to $22.45 \pm 6.09$ $\mathrm{ng} / \mathrm{mL}$ in pre-pubertal goats (Figure 2A). There were no significant fluctuations in INSL3 levels during the tested period, except the increase $(\mathrm{P}<0.05)$ observed from week $20(13.62 \pm 3.25) \mathrm{ng} / \mathrm{ml}$ to $22(22.45 \pm 6.09$ $\mathrm{ng} / \mathrm{mL}$ ) of age.

The detection range of the testosterone assay was 0.01 to $40 \mathrm{ng} / \mathrm{mL}$. The intra-assay coefficient of variations was $3.72 \%(n=6)$ for testosterone. Testosterone concentrations ranged from $0.30 \pm 0.07$ to $1.22 \pm 0.43 \mathrm{ng} / \mathrm{ml}$ in pre-pubertal goats and did not differ from 19 to 22 weeks of age (Figure 2B). Interestingly, the testosterone concentration dropped $(\mathrm{P}<0.05)$ on week 23 and increased $(\mathrm{P}<0.05)$ by more than three-fold by week 28.

There was a significant correlation $(\mathrm{r}=0.58 ; \mathrm{P}<0.05)$ between INSL3 concentration and scrotal circumference (ranged from 7.5 to $18.0 \mathrm{~cm}$ ) in male pre-pubertal goats from 19 to 28 weeks of age. Correlations among testosterone concentration and scrotal circumference, INSL3 concentration and testosterone concentration, each hormone concentration and body weight were statistically non-significant. Body weight was increased with age from week 19 to 28 (Figure 3). Although a 23.4\% increase in scrotal circumference was noted during the study period, it was not significant ( $\mathrm{P}>0.05$; Figure 3 ). Obviously, the scrotal circumference and the body weight (ranged from 8.5 to $19.9 \mathrm{~kg}$ ) was correlated $(\mathrm{r}=0.65 ; \mathrm{P}<0.05)$ during the measured period.
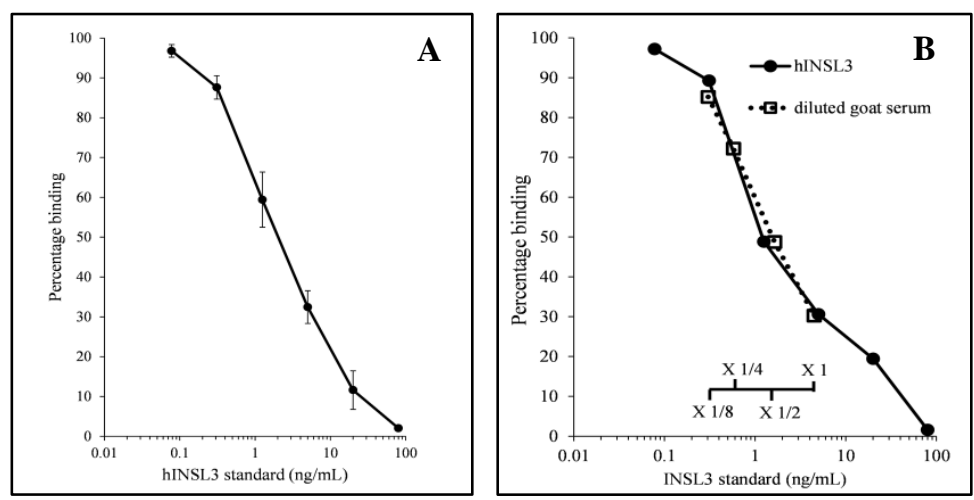

Figure 1. (A) Calibration curve of the enzyme immunoassay for INSL3 showing the reliable detection range, 0.08-80 ng/ml. Mean percentage B/BO \pm SEM of 3 curves. (B) Parallelism for human INSL3 standards with two- to eight-fold serial dilutions of the serum sample from a male goat. Standards for human INSL3 ranged from 0.08 to $80 \mathrm{ng} / \mathrm{ml}$. hINSL3, human INSL3. 

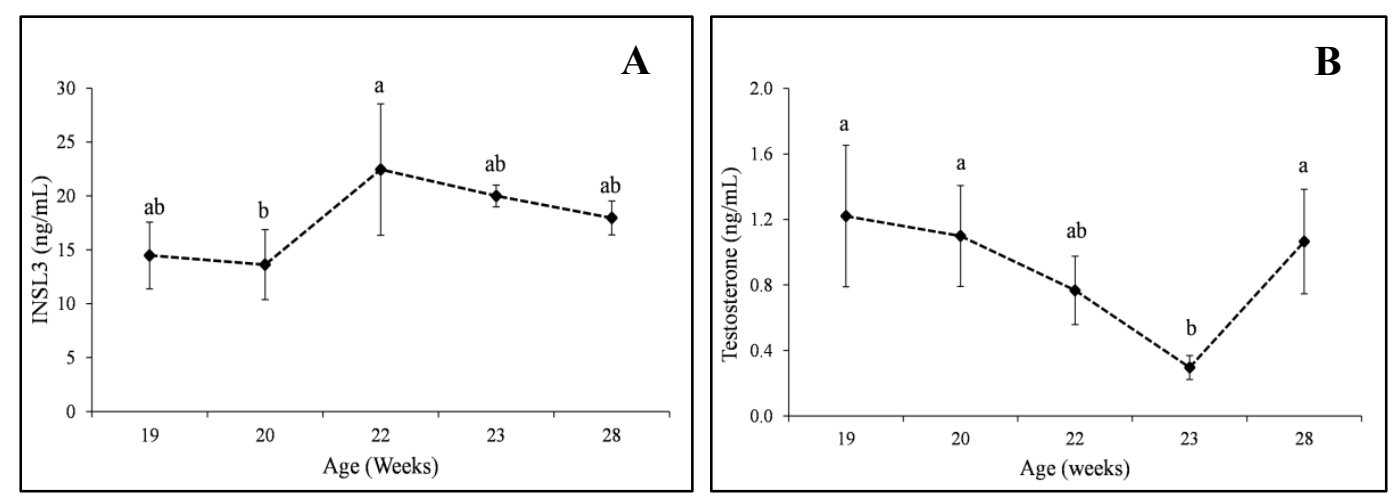

Figure 2. Mean \pm SEM serum concentrations of INSL3 $(A)$ and testosterone $(B)$ in pre-pubertal goats $(n=$ 6 at each week). ${ }^{a-b}$ within a hormone, means without a common superscript differs $(P<0.05)$.

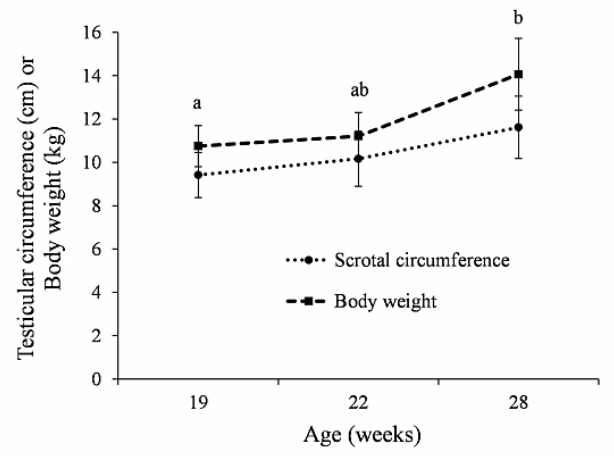

Figure 3. Scrotal circumference and body weight change in goats from 19 to 28 weeks of age. Data are expressed as mean \pm SEM $\left(n=6\right.$ at each week). ${ }^{a^{-}-b}$ Different superscripts indicate the significant difference for mean body weight at each week $(P<0.05)$.

\section{Discussion}

The EIA is a simple, sensitive and specific analytical tool once hormone measurements are considered. However, expensive equipment limits its applications especially in developing world. The present paper reports a simplified version of the existing EIA procedure (Hannan et al., 2016) to measure INSL3 in goats. The established EIA procedure excluded the previously reported INSL3 extraction procedure using a vacuum centrifugation and reduced the assay duration by more than $3 \mathrm{~h}$. The present EIA system showed an improved minimum detection limit of $0.08 \mathrm{ng} / \mathrm{mL}$ and maintained a broad INSL3 detection range of 0.08 to $80 \mathrm{ng} / \mathrm{mL}$. The minimum detection limit and the detection range of first reported INSL3 EIA were $0.31 \mathrm{ng} / \mathrm{mL}$ and 0.31 to $20 \mathrm{ng} / \mathrm{mL}$, respectively (Hannan et al., 2016). The minimum detection limit of the present EIA was almost similar to recently reported time-resolved fluorescence immunoassay (TRFIA) which showed an improved sensitivity (Hannan et al., 2017a).

To the best of our knowledge, there are no previous reports on INSL3 measurements in goats reared in the tropics. The circulating INSL3 levels have only been published in a native Japanese miniature goat breed, Shiba (Hannan et al., 2016; Hannan et al., 2017a; Hannan et al., 2017b). The serum INSL3 concentrations reported in the present study were quite comparable to that of Shiba goats of the same age. The INSL3 concentrations of Jamnapari $\times$ local crossbred goats did not differ much during the tested period, except the significant increase observed from 20 to 22 weeks. In contrast, INSL3 concentrations have been increased approximately in two times from 18 to 28 weeks in Shiba goats (Hannan et al., 2017a). The variation in INSL3 concentrations between two studies is possibly due to the difference in breeds and in management practices. The goats of the former study had been managed for experimental purposes inside an experimental room, while the goats of the present study were managed under routine farm management procedures. Possibly, the process of sexual maturity varied under the two management conditions, especially with feeding management.

The pattern of testosterone secretion was different from that of INSL3 during the study period. A marked drop (three- to four-fold) in testosterone concentrations was observed at 23 weeks compared with weeks 19, 20 and 28. Several previous studies have reported similar observations in Shiba goats after 20 weeks (Tani et al., 1992) 
and 26 weeks (Hannan et al., 2017a) of age, and also in Black Bengal goats during 12 to 20 weeks (Georgie et $a l ., 1985)$ of age. It is understood that testosterone is acutely regulated by the hypothalamic-pituitary-gonadal (HPG) axis and subjected to acute episodic fluctuations of LH. Especially, the HPG axis is activated during puberty. We have recently showed that INSL3 is also acutely regulated by LH in goats (Hannan et al., 2016); however, the amplitude of INSL3 rise is much lower than that of testosterone.

In the present study, a significant positive correlation was observed between serum INSL3 concentrations and the testicular circumference of Jamnapari $\times$ local crossbred goats from 19 to 28 weeks of age. Previously, Hannan et al. (Hannan et al., 2017a) has also shown a strong positive correlation between the plasma concentrations of INSL3 and the scrotal circumference in Shiba goats. In humans, increasing INSL3 concentrations were associated with increasing testicular volume during pubertal development (Ferlin et al., 2006). In contrast to INSL3 concentrations, testosterone concentrations were not significantly correlated with testicular circumference in tested goats in the present study. In line with this observation, a weak correlation has been observed between plasma testosterone and testicular circumference in Shiba goats (Hannan et al., 2017a). It has been argued that circulating INSL3 concentrations better reflect the status of Leydig cells than testosterone concentrations, when single time-point blood samples are considered (Hannan et al., 2017a). The acute dependence of testosterone secretions on pulsatile secretions of LH regulated by HPG axis, compared with INSL3 secretions, may also be critical in this scenario.

\section{Conclusions}

A rapid and highly sensitive enzyme immunoassay was developed to quantify INSL3 in goats by modifying an existing EIA procedure. Different serum INSL3 and testosterone dynamics were found during 19 to 28 weeks of age. The scrotal circumference was significantly correlated with INSL3 concentrations. Compared with testosterone concentrations, INSL3 concentrations seemed to be more consistent with the growth of pre-pubertal goats. Further studies in a broad age range with more frequent sampling would be needed to confirm these results in tropical goat breeds.

\section{Acknowledgements}

The authors are grateful to Dr. Erika E. Büllesbach, Medical University of South Carolina, USA for providing anti-bovine INSL3 antibody (2-8F).

\section{Conflict of interest}

None to declare.

\section{References}

Anand-Ivell R, K Heng, B Hafen, B Setchell and R Ivell, 2009. Dynamics of INSL3 peptide expression in the rodent testis. Biol. Reprod., 81: 480-487.

Anand-Ivell R, J Wohlgemuth, MT Haren, PJ Hope, G Hatzinikolas, G Wittert and R Ivell, 2006. Peripheral INSL3 concentrations decline with age in a large population of Australian men. Int. J. Androl., 29: 618-626.

Bay K and AM Andersson, 2011. Human testicular insulin-like factor 3: in relation to development, reproductive hormones and andrological disorders. Int. J. Androl., 34: 97-109.

Bay K, S Hartung, R Ivell, M Schumacher, D Jurgensen, N Jorgensen, M Holm, NE Skakkebaek and AM Andersson, 2005. Insulin-like factor 3 serum levels in 135 normal men and 85 men with testicular disorders: relationship to the luteinizing hormone-testosterone axis. J. Clin. Endocrinol. Metab., 90: 3410-3418.

Bay K, HE Virtanen, S Hartung, R Ivell, KM Main, NE Skakkebaek, AM Andersson, G Nordic Cryptorchidism Study and J Toppari, 2007. Insulin-like factor 3 levels in cord blood and serum from children: effects of age, postnatal hypothalamic-pituitary-gonadal axis activation, and cryptorchidism. J. Clin. Endocrinol. Metab., 92: 4020-4027.

Bullesbach EE, R Rhodes, B Rembiesa and C Schwabe, 1999. The relaxin-like factor is a hormone. Endocrine, 10: 167-169.

Bullesbach EE and C Schwabe, 2002. The primary structure and the disulfide links of the bovine relaxin-like factor (RLF). Biochemistry, 41: 274-281.

Ferlin A, A Garolla, F Rigon, L Rasi Caldogno, A Lenzi and C Foresta, 2006. Changes in serum insulin-like factor 3 during normal male puberty. J. Clin. Endocrinol. Metab., 91: 3426-3431.

Georgie G, S Mehta, V Dixit, B Sengupta and A Kanaujia, 1985. Peripheral plasma testosterone levels in two Indian breeds of goats and their reciprocal crosses. Anim. Reprod. Sci., 9: 95-98. 
Hannan MA, Y Fukami, N Kawate, M Sakase, M Fukushima, IN Pathirana, EE Bullesbach, T Inaba and H Tamada, 2015. Plasma insulin-like peptide 3 concentrations are acutely regulated by luteinizing hormone in pubertal Japanese Black beef bulls. Theriogenology, 84: 1530-1535.

Hannan MA, N Kawate, Y Fukami, IN Pathirana, EE Bullesbach, T Inaba and H Tamada, 2016. Acute regulation of plasma insulin-like peptide 3 concentrations by luteinizing hormone in male goats. Theriogenology, 86: 749-756.

Hannan MA, N Kawate, Y Fukami, W Weerakoon, EE Bullesbach, T Inaba and H Tamada, 2017a. Changes of plasma concentrations of insulin-like peptide 3 and testosterone, and their association with scrotal circumference during pubertal development in male goats. Theriogenology, 92: 51-56.

Hannan MA, N Kawate, Y Fukami, WW Weerakoon, EE Bullesbach, T Inaba and H Tamada, 2017b. Effects of long-acting GnRH antagonist, degarelix acetate, on plasma insulin-like peptide 3, testosterone and luteinizing hormone concentrations, and scrotal circumference in male goats. Theriogenology, 88: 228-235.

Ivell R, JD Wade and R Anand-Ivell, 2013. INSL3 as a biomarker of Leydig cell functionality. Biol. Reprod., 88: 147, 141-148.

Johansen ML, R Anand-Ivell, A Mouritsen, CP Hagen, MG Mieritz, T Soeborg, TH Johannsen, KM Main, AM Andersson, R Ivell and A Juul, 2014. Serum levels of insulin-like factor 3, anti-Mullerian hormone, inhibin $\mathrm{B}$, and testosterone during pubertal transition in healthy boys: a longitudinal pilot study. Reproduction, 147: 529-535.

Kawamura K, J Kumagai, S Sudo, S-Y Chun, M Pisarska, H Morita, J Toppari, P Fu, JD Wade and RA Bathgate, 2004. Paracrine regulation of mammalian oocyte maturation and male germ cell survival. Proc. Natl. Acad. Sci. U.S.A., 101: 7323-7328.

Kawate N, A Ohnari, IN Pathirana, M Sakase, EE Bullesbach, M Takahashi, T Inaba and H Tamada, 2011. Changes in plasma concentrations of insulin-like peptide 3 and testosterone from birth to pubertal age in beef bulls. Theriogenology, 76: 1632-1638.

Nef S and LF Parada, 1999. Cryptorchidism in mice mutant for Insl3. Nat. Genet., 22: 295-299.

Pathirana IN, H Yamasaki, N Kawate, M Tsuji, EE Bullesbach, M Takahashi, S Hatoya, T Inaba and H Tamada, 2012. Plasma insulin-like peptide 3 and testosterone concentrations in male dogs: changes with age and effects of cryptorchidism. Theriogenology, 77: 550-557.

Siqin, I Minagawa, M Okuno, K Yamada, Y Sugawara, Y Nagura, K-I Hamano, EY Park, H Sasada and T Kohsaka, 2013. The active form of goat insulin-like peptide 3 (INSL3) is a single-chain structure comprising three domains BCA, constitutively expressed and secreted by testicular Leydig cells. Biol. Chem., 394: 1181-1194.

Tani M, T Sawada, T Ishigami, M Kishimoto and J Mori, 1992. Androstenedione and Testosterone Levels in Peripheral Plasma of Male Shiba Goats during Development. J. Reprod. Dev., 38: 235-238.

Wikstrom AM, K Bay, M Hero, AM Andersson and L Dunkel, 2006. Serum insulin-like factor 3 levels during puberty in healthy boys and boys with Klinefelter syndrome. J. Clin. Endocrinol. Metab., 91: 4705-4708.

Zimmermann S, G Steding, JM Emmen, AO Brinkmann, K Nayernia, AF Holstein, W Engel and IM Adham, 1999. Targeted disruption of the Insl3 gene causes bilateral cryptorchidism. Mol. Endocrinol., 13: 681-691. 